EUROPEAN ORGANIZATION FOR NUCLEAR RESEARCH

European Laboratory for Particle Physics

Large Hadron Collider Project

LHC Project Report 343

\title{
LOCALIZATION OF ELECTRICAL INSULATION FAILURES \\ IN SUPERCONDUCTING COLLARED COILS BY ANALYSIS \\ OF THE DISTORTION OF A PULSED MAGNETIC FIELD
}

Piotr Komorowski ${ }^{1}$ and Davide Tommasini ${ }^{2}$

\begin{abstract}
The localization of possible electrical faults in superconducting accelerator magnets may, in most cases, be a complex, expensive and time-consuming process. In particular, inter-turn short circuits and failures of the ground insulation are well detectable when the magnet is collared, but often disappear after disassembly for repair due to the release of the pre-stress in the coils.

The fault localization method presented in this paper is based on the measurement and analysis of the magnetic field generated inside the magnet aperture by a high voltage pulse. The presence of the fault modifies the distribution of the current in the coils and produces a distortion of the magnetic field. The described method aims at locating both the longitudinal and azimuthal position of the fault-affected area. The test method, the transient case FEM models and the implemented experimental set-up are presented and discussed for the LHC dipole models.
\end{abstract}

1 Faculty of Physics, Warsaw University of Technology, Warsaw, Poland

2 LHC Division, CERN

Presented at the 16th International Conference on Magnetic Technology

26 September-2 October 1999 - Ponte Vedra Beach, USA

Administrative Secretariat

LHC Division

CERN

CH - 1211 Geneva 23

Switzerland

Geneva, 1 December 1999 


\title{
Localization of Electrical Insulation Failures in Superconducting Collared Coils by Analysis of the Distortion of a Pulsed Magnetic Field
}

\author{
Piotr Komorowski ${ }^{*}$, Davide Tommasini \\ CERN, LHC Division, Geneva, Switzerland \\ *Faculty of Physics, Warsaw University of Technology, Warsaw, Poland
}

\begin{abstract}
The localization of possible electrical faults in superconducting accelerator magnets may, in most cases, be a complex, expensive and time-consuming process. In particular, inter-turn short circuits and failures of the ground insulation are well detectable when the magnet is collared, but often disappear after disassembly for repair due to the release of the pre-stress in the coils.

The fault localization method presented in this paper is based on the measurement and analysis of the magnetic field generated inside the magnet aperture by a high voltage pulse. The presence of the fault modifies the distribution of the current in the coils and produces a distortion of the magnetic field. The described method aims at locating both the longitudinal and azimuthal position of the fault-affected area. The test method, the transient case FEM models and the implemented experimental set-up are presented and discussed for the LHC dipole models.
\end{abstract}

\section{INTRODUCTION}

The pattern of the magnetic field in modern superconducting accelerator magnets is mainly determined by the geometry of the coils. In an ideal case a current of the type $\mathrm{I}(\varphi)=\mathrm{I}_{0} \cos (\varphi)$ distributed around the aperture produces a pure dipole field. For practical reasons multilayer coils graded with longitudinal wedge-shaped spacers inserted between the conductor blocks approximate this condition (Fig. 1). This paper is focussed on the study of the single-aperture superconducting short dipole models for the LHC in view of applying the results of the investigation to the 15-m long double-aperture magnets.

Since 1989 a test and evaluation program of short superconducting dipole magnets for the LHC is under way at CERN. It was intensified in 1995 when a production of a new series of 1-m long dipole magnets was launched [1]. The design is based on a dismountable structure allowing easy implementation of variants (Fig. 2). The coils are confined in a fixed volume by the collars with a pre-

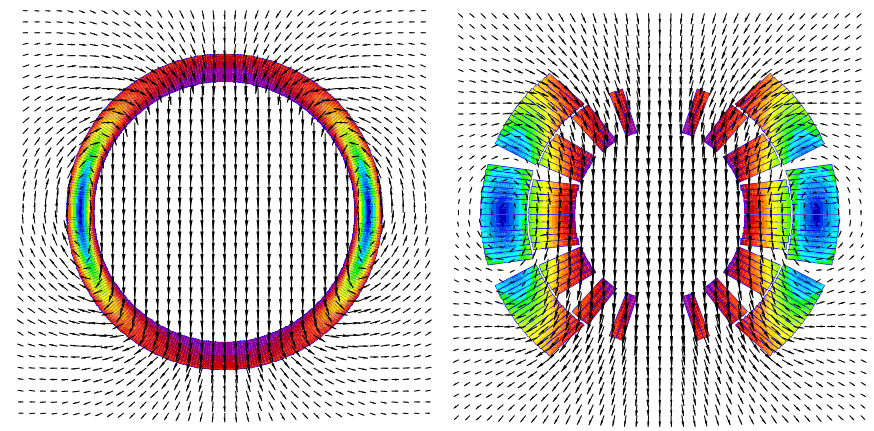

Fig. 1. Ideal current shell vs. actual LHC dipole geometry

Manuscript received September 27, 1999.

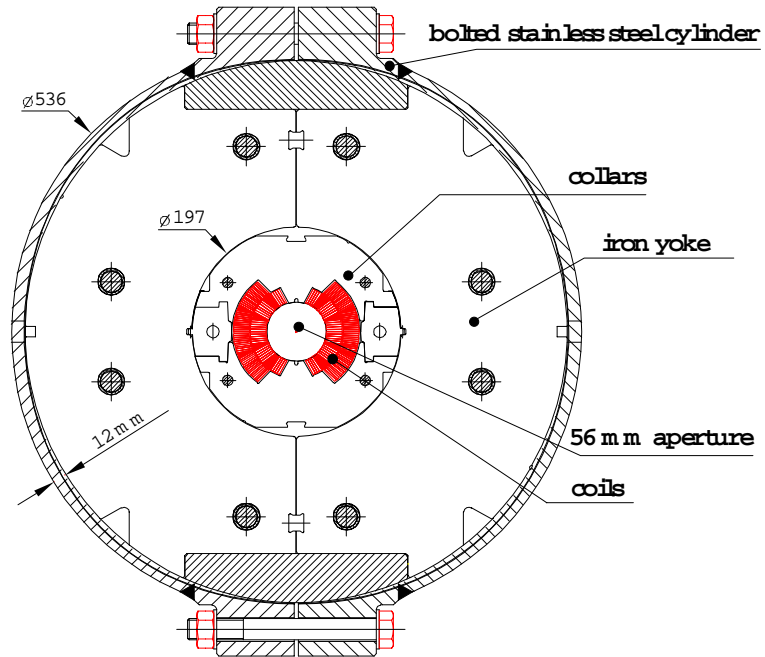

Fig. 2. Cross section of a single-aperture dipole magnet

compression sufficient to counteract the electromagnetic forces during magnet excitation. The outer layer of the coils is separated from the collars by $0.5 \mathrm{~mm}$ of ground insulation and by a $0.7-\mathrm{mm}$ thick austenitic steel protection sheet called 'collaring shoe'. A ferromagnetic yoke is clamped around the collared assembly by an external bolted stainless steel cylinder. The yoke enhances the magnitude of the magnetic field induction inside the aperture and prevents the collars from expanding when submitted to the Lorentz forces exerted on the coils.

The superconducting cable is insulated by two layers of polyimide film followed by a third adhesive one, which bonds adjacent turns of the coil. The effective thickness of the cable insulation is about $125 \mu \mathrm{m}$; i.e. two adjacent turns are separated by about $250 \mu \mathrm{m}$ of insulation. This dielectric barrier can be damaged during one of the magnet assembly phases, causing thus an inter-turn short circuit. The change in the distribution of the current in the coils of the magnet due to such a failure produces a distortion of the magnetic field. This effect is used here for the localization of the short circuits by means of transient magnetic field analysis.

\section{INTER-TURN SHORT-CIRCUITS}

Inter-turn short circuits are well detectable when the magnet is collared. The presence of the insulation failure in the magnet can be diagnosed by means of pulse propagation methods at room temperature. A voltage impulse is produced between the terminals of the magnet by a discharge generator. The coil response in form of voltage and current oscillations is then recorded and analyzed in terms of pseudo-period of the oscillations, inductance and effective resistance of the coils. Fig. 3 shows the discharge curves acquired on the collared assembly during a test at $1 \mathrm{kV}$. 


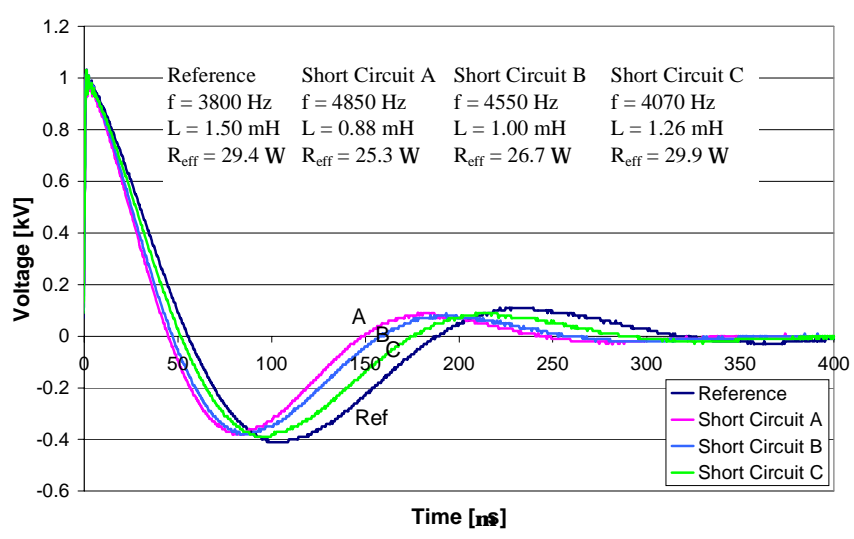

Fig. 3. Discharge curves at $1 \mathrm{kV}$

In case of an inter-turn short circuit, the current is redirected to the neighboring cable turn through the shortcircuit path (Fig. 4). As a consequence of this redistribution a high current is induced in the passive winding deprived of the initially imposed transport current. This induced current generates a transient magnetic field opposing the flux change in the area spawn by the fault-affected loop (schematically marked with a thin line in Fig. 4.) Energy is dissipated additionally at the point of fault. The value of the magnet inductance decreases, whereas the damping factor and the frequency of the oscillations increase. The discharge curves already give a valuable clue concerning the azimuthal position of the defect, i.e. the bigger the frequency change, and the greater the self-inductance drop, the closer the failure to the median plane of the magnet (see Fig. 3 and 4). The existence of the electrical failure can be thus verified, however its longitudinal position cannot be established. The analysis of the transient magnetic field during the discharge provides a solution to this problem, as demonstrated in the following sections.

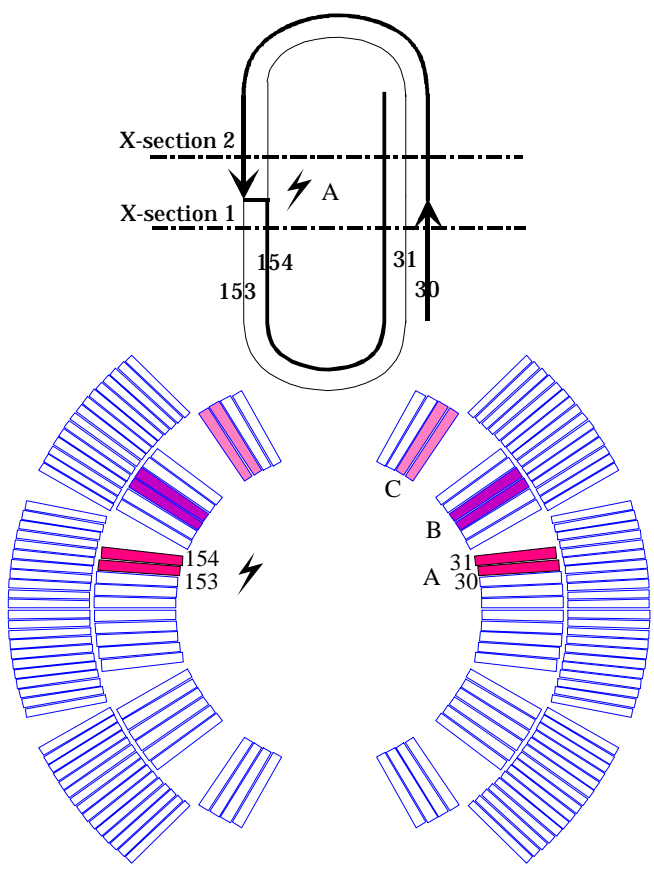

Fig. 4. Schematic representation of an inter-turn short-circuit

\section{MAGNET MODELING}

\section{A. Multipole Expansion of the Magnetic Field}

The radial and azimuthal components of the magnetic field can be expressed as:

$$
\begin{aligned}
& B_{r}(r, \theta)=B_{1} \sum_{n=1}^{\infty}\left(\frac{r}{r_{0}}\right)^{n-1}\left(b_{n} \sin n \theta+a_{n} \cos n \theta\right) \\
& B_{\theta}(r, \theta)=B_{1} \sum_{n=1}^{\infty}\left(\frac{r}{r_{0}}\right)^{n-1}\left(b_{n} \cos n \theta-a_{n} \sin n \theta\right)
\end{aligned}
$$

Coefficients $b_{\mathrm{n}}$ and $a_{\mathrm{n}}$ indicate respectively the normal and the skew field multipoles measured at a reference radius $r_{0}$ and normalized to the main dipole field $B_{1}$. The main normal coefficient of the multipole expansion $\left(b_{1}\right)$ is thus always normalized to 1 . Higher harmonic coefficients represent magnetic field errors relative to the dipole $B_{1}$ and are given in dimensionless units of $10^{-4}$.

\section{B. Reference Magnet Models}

Both static and transient models of the 5-block collared dipole magnet ( 2 conductor blocks in the outer, and 3 in the inner layer of the coil quadrant) without iron yoke were developed and analyzed using two different CAD programs. In the static model the conductors are excited with a $20 \mathrm{~A}$ direct current. This model was created with The Routine for the Optimization of Magnet X-Sections, Inverse Problem Solving and End Region Design (ROXIE) program package developed at CERN [2], and used for the integrated design of superconducting accelerator magnets. The geometry of the coils defined in ROXIE served as a reference for the transient model implemented in OPERA-2D [3]. Table I contains a geometry transfer cross-check between the two CAD environments.

TABLE I

COMPARISON OF STATIC MODELS AT $R_{0}=10 \mathrm{MM}$

\begin{tabular}{lllll}
\hline Static Case & $\mathrm{B}_{1}\left[\cdot 10^{-4} \mathrm{~T}\right]$ & $\mathrm{b}_{3}$ & $\mathrm{~b}_{5}$ & $\mathrm{~b}_{7}$ \\
\hline ROXIE & -122.2 & -0.52 & 0.017 & 0.013 \\
OPERA-2D & -119.3 & -0.55 & 0.020 & 0.013 \\
\hline
\end{tabular}

The transient model uses a FEM approach to simulate the behavior of the magnet during a $1 \mathrm{kV}$ pulsed discharge in which the peak current reaches a value of $20 \mathrm{~A}$. In this case the voltage-current decay, the skin, eddy currents and proximity effects in the conductors, copper wedges, collaring shoe, and in the collars are taken into account. The effective conductivity of the collars was adjusted in the model in order to fine-tune the computational results to the experimental data acquired by measuring the peak field induction inside the aperture and in several locations around the collars. For setting the correct value of the conductivity of the cables an additional test was carried out to evaluate the magnitude of the eddy current effects. For this purpose separate layers of the coils were excited one at a time to analyze the screening effects. The parameters of the model have been optimized until the simulated magnetic field map inside and outside the magnet matched the experimental results. 
IV. Transient Magnetic Field in PResence OF A ShortCIRCUIT

TABLE II

SHORT-CIRCUIT ANALYSIS AT $R_{0}=10 \mathrm{MM}$

\begin{tabular}{|c|c|c|c|}
\hline Normal & Reference & $\mathrm{X}$-section 1 & X-section 2 \\
\hline $\mathrm{B}_{1}$ & $-82.2 \cdot 10^{-4} \mathrm{~T}$ & $-60.9 \cdot 10^{-4} \mathrm{~T}$ & $-61.4 \cdot 10^{-4} \mathrm{~T}$ \\
\hline$b_{2}$ & 0.0 & 113.2 & 0.0 \\
\hline$b_{3}$ & -156.4 & -768.4 & -697.0 \\
\hline $\mathrm{b}_{4}$ & 0 & 31.6 & 0.0 \\
\hline$b_{5}$ & 9.8 & -9.9 & 2.8 \\
\hline$b_{6}$ & 0.0 & 5.2 & 0.0 \\
\hline $\mathrm{b}_{7}$ & 0.3 & 1.4 & 3.1 \\
\hline Skew & Reference & $\mathrm{X}$-section1 & $\mathrm{X}$-section 2 \\
\hline$a_{1}$ & 0.0 & 271.5 & 0.0 \\
\hline$a_{2}$ & 0.0 & 1320.9 & 1455.7 \\
\hline$a_{3}$ & 0.0 & 45.8 & 0.0 \\
\hline$a_{4}$ & 0.0 & 195.0 & 204.5 \\
\hline$a_{5}$ & 0.0 & 1.0 & 0.0 \\
\hline$a_{6}$ & 0.0 & 23.5 & 22.7 \\
\hline$a_{7}$ & 0.0 & -0.7 & 0.0 \\
\hline
\end{tabular}

In case of short-circuit the transport current follows a path different from the nominal one (Fig. 4). On one side of the inter-turn short-circuit the current distribution in the coil windings is asymmetric (cross-section 1). On the other side it becomes symmetric again (cross-section 2). In terms of the magnetic field quality this corresponds to the vanishing of even-order harmonics of the normal field and of odd orders of the skew multipoles in the area where the current distribution is symmetric. Table II lists the computed multipoles values for the short-circuit in position denoted as $A$ (Fig. 4) in the two sections before and after the defect in comparison to the pulsed magnetic field generated in a flawless magnet.

\section{EXPERIMENTAL SET-UP}

\section{A. Detection Method}

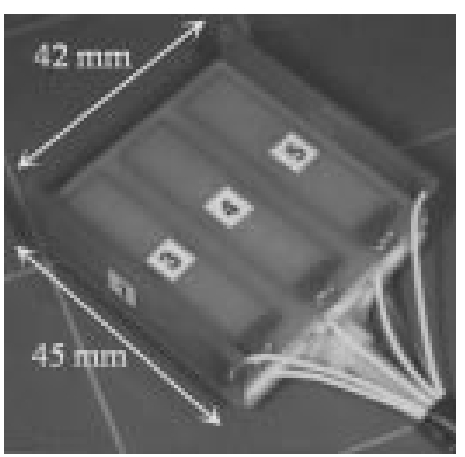

The development of the field harmonics during a pulsed discharge is detected with a system of pick-up coils (Fig. 5) aligned on the median plane of the magnet. The schematic position of the individual pick-up coils inside the aperture is shown in Fig. 6. In order to visualize and explain the detection method the pure normal and skew up coils

quadrupoles in Fig. 6 were chosen as the representatives of the even-order field harmonics, and the pure normal and skew sextupoles as the representatives of the odd-order multipoles. If the even orders of the normal field harmonics are present in the magnetic field a difference between the voltage induced in coils $\mathrm{C} 3$ and $\mathrm{C} 5$ will be observed. The contribution of the even-order multipoles cancels out in the central coil. Since higher order odd multipoles are negligible close to the center of the aperture, coil $\mathrm{C} 4$ picks up mainly the dipole field. Altogether coils C3, C4 and C5 are transparent to the flux resulting from the skew harmonics, which is detected by the coils $\mathrm{C} 1$ and $\mathrm{C} 2$. The sum of the voltage induction on these two coils will correspond to twice the total of the odd-order skew multipoles.
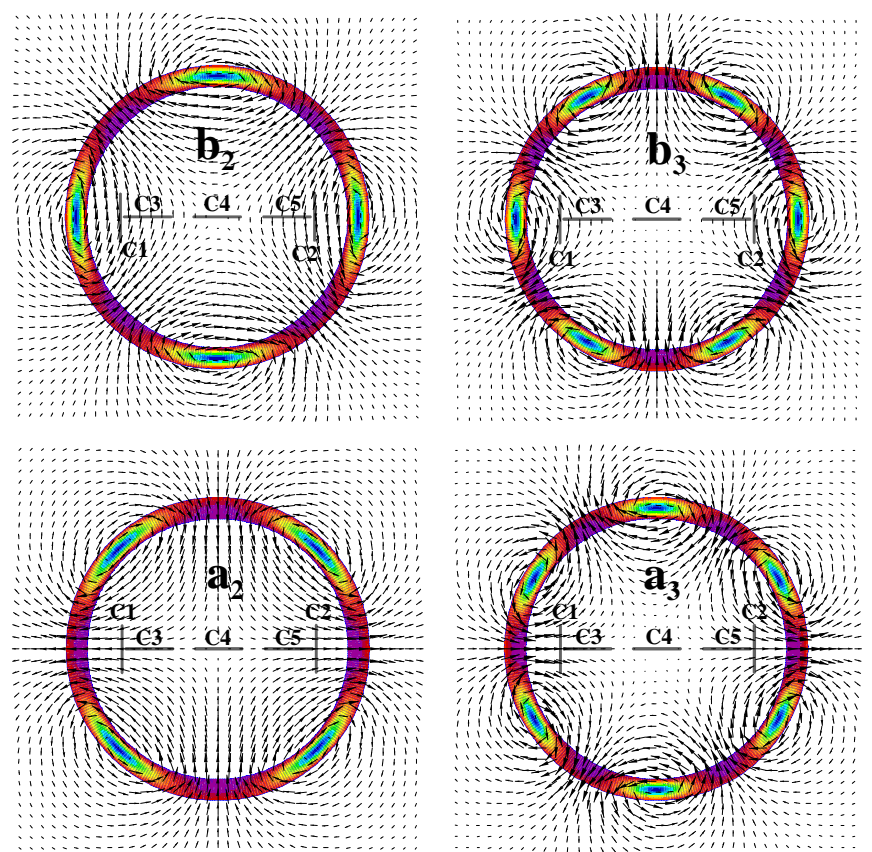

Fig. 6. Schematic representation of the detection method

\section{B. Test Set-up}

One of the short dipole magnets was equipped with micro-relays soldered on the internal layers in two clusters; one centered around the cross section in the middle of the magnet (Fig. 7), and the other close to the end region of the coil. The relays, excited externally with DC one at a time, create short circuits between adjacent cable turns in well-defined positions. A pulsed current is supplied from a discharge generator by releasing the energy accumulated in a $1 \mu \mathrm{F}$ capacitor, charged at

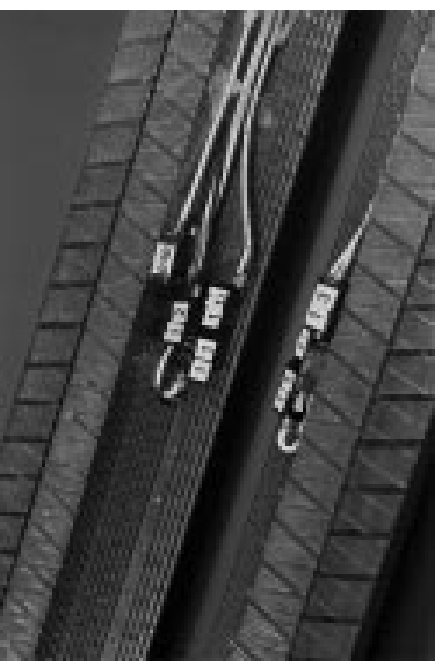

Fig. 7. Magnet coils equipped with micro-relays $1 \mathrm{kV}$, into the magnet. The voltage induced in the pick-up coils is measured during the discharge and integrated thereafter to obtain the time evolution of the magnetic field induction. The pick-up coil platform slides longitudinally on two vetronite rods aligned inside the aperture of the magnet. 


\section{Results}

The test results confirm the computational analysis demonstrated in previous sections. The signals registered by the five pick-up coils of Fig. 5 allowed to identify the longitudinal position of all triggered faults, and also gave an indication of the azimuthal position of the short-circuits. As an example, typical results of the measurements are presented in Fig. 8 for a short-circuit in position A. As expected, the signals of the absolute peak field induction on
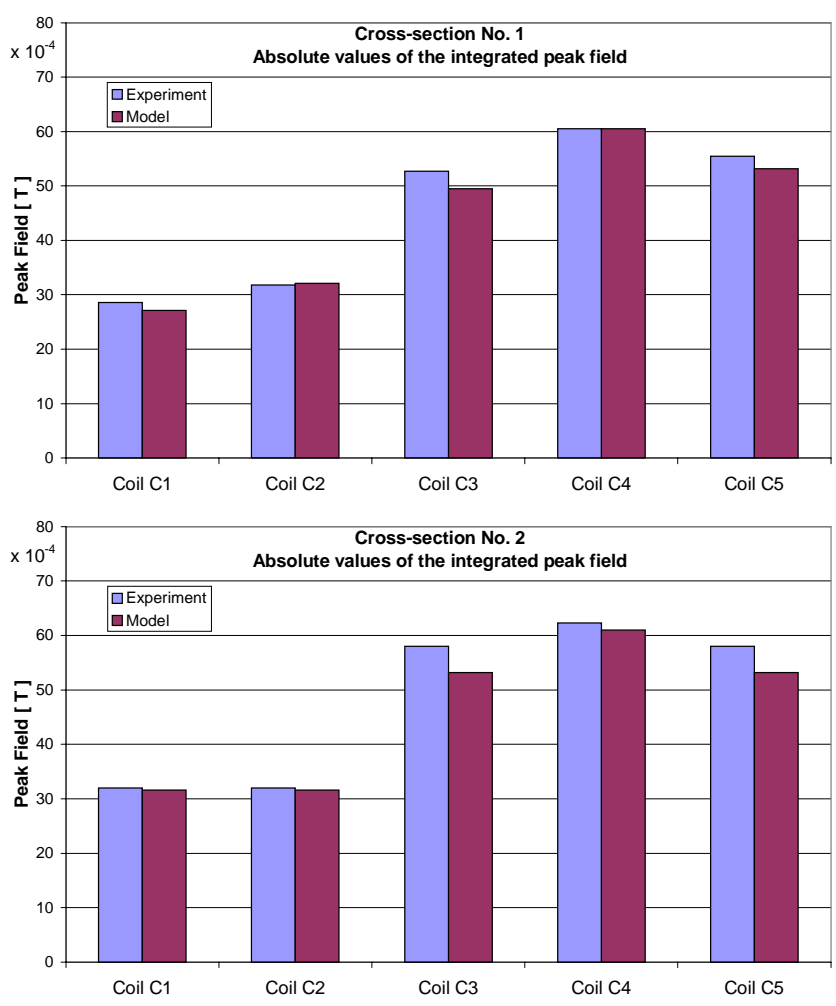

Fig. 8. Test results for short circuit A. In reality the signals picked up by the coils $\mathrm{C} 1$ and $\mathrm{C} 2$ are of opposite polarity.

the coils $\mathrm{C} 1$ and $\mathrm{C} 2$, as well as on the coils $\mathrm{C} 3$ and C5 became unbalanced while passing the point of fault from cross-section No. 2 towards cross-section No. 1. The high magnitude of the signal on the coils $\mathrm{C} 1, \mathrm{C} 2$ is due to the high current induced in the passive winding loop deprived of the imposed transport current. For the short circuit under analysis the peak intensity of this current amounts to

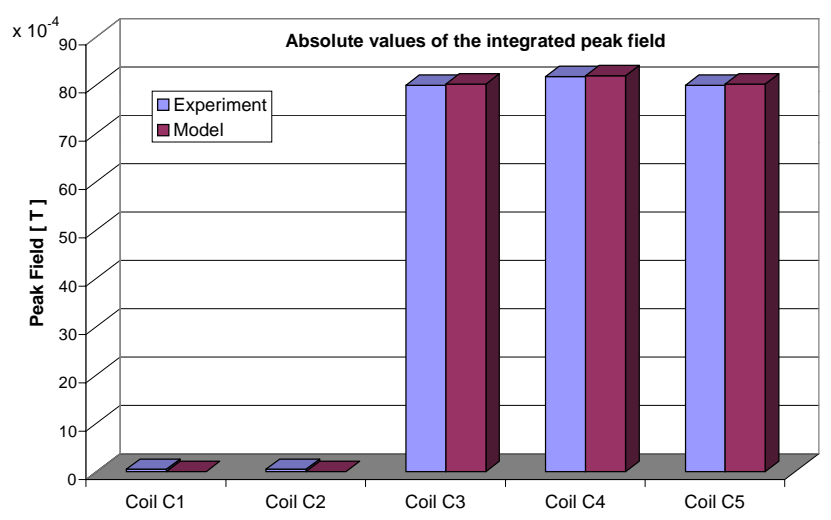

Fig. 9. Pick-up coil indications in a flawless magnet

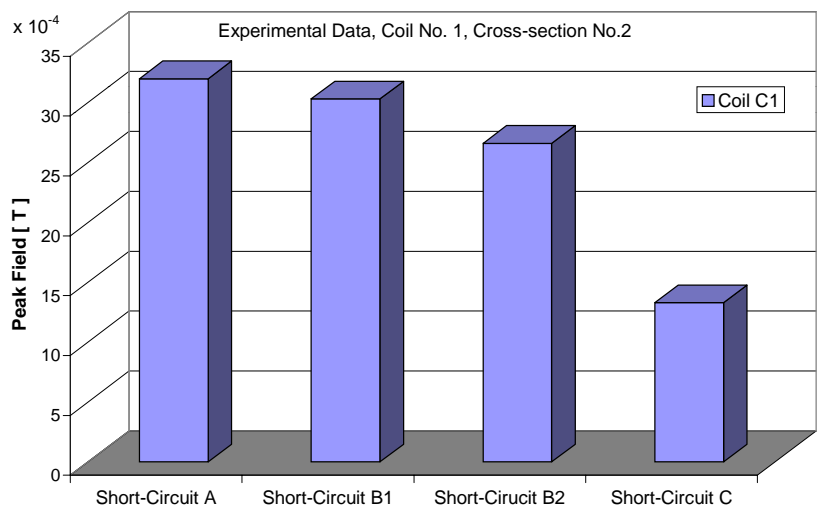

Fig. 10. Indications of the coil $\mathrm{C} 1$ as a function of the azimuthal position of short-circuit

approximately $510 \mathrm{~A}$. In case of the flawless magnet with no short-circuits engaged, virtually no signal is induced in the coils $\mathrm{C} 1$ and $\mathrm{C} 2$ (Fig. 9).

Also, as predicted by the model, the amplitude of the signal picked up by the coils $\mathrm{C} 1$ and $\mathrm{C} 2$ is very sensitive to the azimuthal position of the short-circuit (Fig. 10). The difference in the coil indications is visible even for the defects located close to each other as shown for the shortcircuits B1 and B2 between two pairs of adjacent cable turns in the proximity of the position B in Fig. 4.

\section{CONCLUSIONS}

Transient magnetic field distortions created by electrical insulation failures in superconducting collared coils were analyzed in view of the localization of possible inter-turn short circuits. The detection method was based on the study of the magnetic field harmonics induced by the current redistribution in the magnet coils. The experimental procedure has been simulated with OPERA-2D and tested on a dedicated dipole magnet equipped with micro-relays capable of activating artificial short circuits in the internal layer of the coils. The longitudinal position of the shortcircuits was successfully established for all of the installed micro-switches with the precision of a few millimeters. The method gave also the indication of the azimuthal position of the defects.

\section{ACKNOWLEDGMENT}

The authors would like to thank N. Siegel and C. Wyss for their constant support and $\mathrm{H}$. Kummer for his contribution to the assembly of the test magnet.

\section{REFERENCES}

[1] N.Andreev et al. "State of the short dipole model program for the LHC", presented at the $6^{\text {th }}$ EPAC, Stockholm, June 22-26, 1998

[2] S. Russenschuck et al., "Integrated design of superconducting accelerator magnets. A case of study of the main quadrupole", The European Physical Journal 1998, AP 1, 93-102

[3] OPERA-2D, Software Environment for Electromagnetic Design, Workstation ver. 7.0, 1998, Vector Fields Ltd., Oxford, England 